\title{
Use of the frozen elephant trunk technique for type A intramural hematoma
}

\author{
Luca Di Marco, Giacomo Murana, Alessandro Leone, Mariafrancesca Fiorentino, Ciro Amodio, \\ Roberto Di Bartolomeo, Davide Pacini \\ Cardiac Surgery Unit, Cardio-Thoracic-Vascular Department, S.Orsola Hospital, University of Bologna, Bologna, Italy \\ Correspondence to: Luca Di Marco, MD, PhD. Cardiac Surgery Unit, Cardio-Thoracic-Vascular Department, S.Orsola Hospital, University of \\ Bologna, Via Massarenti 9, 40138 Bologna, Italy. Email: ludima08@libero.it.
}

Submitted May 19, 2019. Accepted for publication Jul 25, 2019.

doi: 10.21037/acs.2019.08.04

View this article at: http://dx.doi.org/10.21037/acs.2019.08.04

\section{Introduction}

Intramural hematoma (IMH) is a well-recognized type of acute aortic syndrome with a significant rate of progression in more than $60 \%$ of cases $(1,2)$.

According to the most recent European guidelines, the treatment of a type A IMH follows the same recommendations as for type $\mathrm{A}$ aortic dissection $(1,3)$. Therefore, in case of aortic arch involvement, a more radical treatment such as frozen elephant trunk (FET) should be considered. The rationale to use this kind of approach is to prepare for a second stage endovascular procedure, especially in case of distal downstream thoracoabdominal aorta involvement and in young patients. In this video, we illustrate the treatment of a type A IMH using the Vascutek Thoraflex hybrid prosthesis.

\section{Clinical vignette}

A 56-year-old woman presented to the Emergency Department with upper back pain, described as tearing, radiating anteriorly and associated with profuse sweating.

She immediately underwent a CT angiogram which showed a type A IMH with a maximum thickness of $5 \mathrm{~mm}$ at the level of the ascending aorta and $10 \mathrm{~mm}$ in the aortic arch.

She was therefore admitted to our department; in the absence of any signs of impending rupture, she was initially treated medically with resolution of chest pain after obtaining good blood pressure control. Serial CT angiogram 72 hours after admission demonstrated partial resorption of the hematoma.

However, one week later, she experienced a new episode of chest pain and the new CT angiogram demonstrated an increased aortic diameter at the isthmus (41 vs. $38 \mathrm{~mm})$ and an increased hematoma-thickness (17 vs. $10 \mathrm{~mm})$ (Figure 1A,B).

After a multidisciplinary evaluation, the possibility of an endovascular treatment was excluded so the patient underwent surgical repair using FET technique, as showed in the following video.

\section{Surgical technique}

\section{Preparation and exposition}

Surgery was performed through full median sternotomy. The innominate vein was identified, interrupted and ligated; then extensive isolation of the arch vessels from the surroundings mediastinal structures was carried out to facilitate later manipulation and anastomosis. After cardiopulmonary bypass (CPB) was instituted through ascending aortic and right atrial cannulation, the ascending aorta was cross-clamped and, after the aortotomy, the cardioplegia was selectively delivered into the coronary ostia.

\section{Operation}

At a nasopharyngeal temperature of 26 degrees Celsius, circulatory arrest was instituted, the aortic clamp removed, the aortic arch opened, and the innominate and left carotid arteries were selectively cannulated in order to start bilateral antegrade cerebral perfusion.

A $28 \mathrm{~mm}$ Thoraflex hybrid graft was used for arch reconstruction and the stent portion was deployed in the 

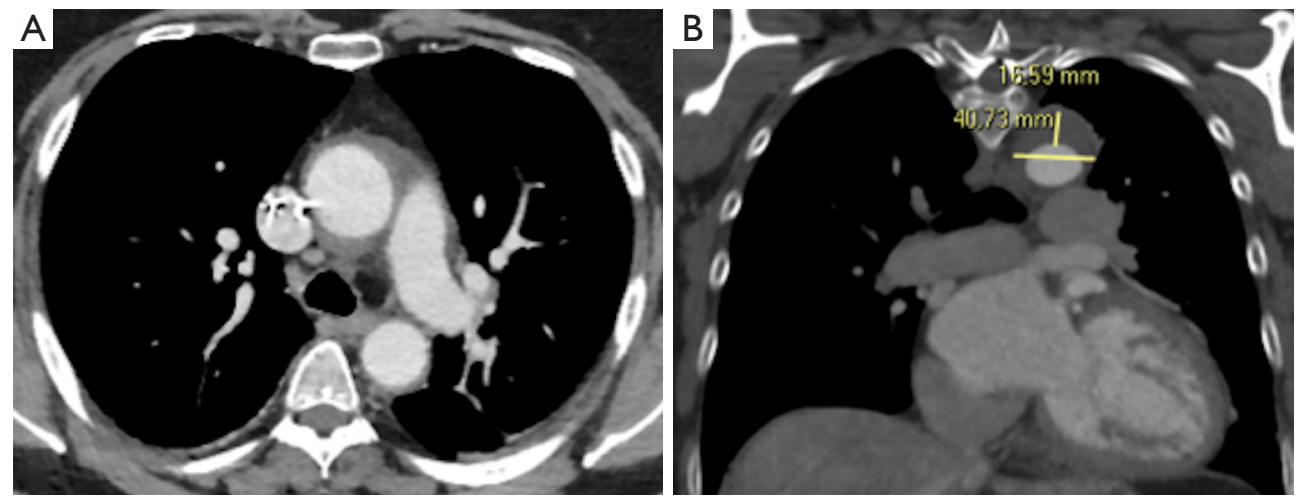

Figure 1 The AngioCT scan showing the IMH of the ascending (A) and descending thoracic aorta (B). IMH, intramural hematoma.
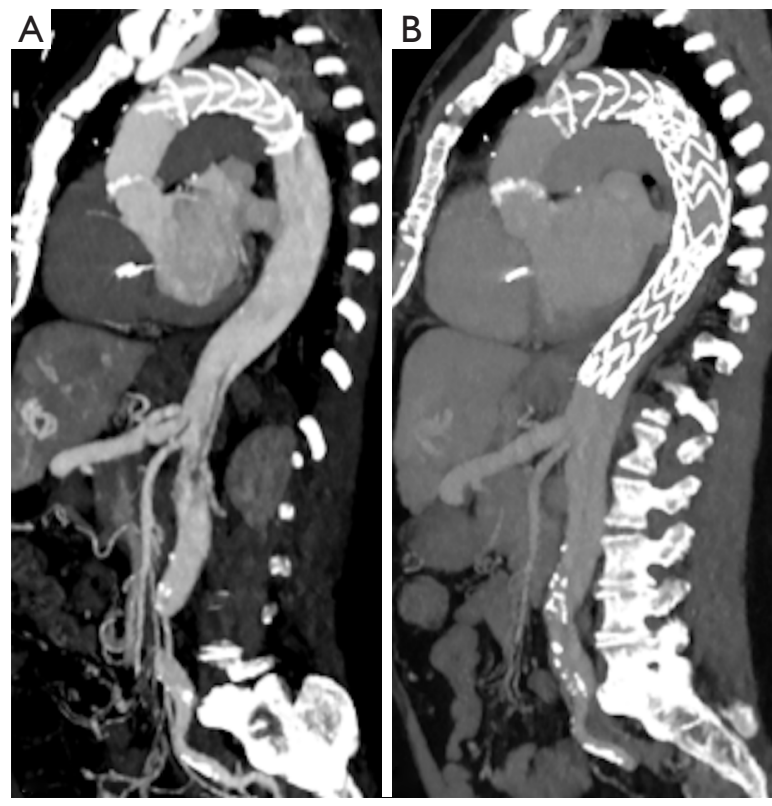

Figure 2 The AngioCT scan after the FET procedure (A) and 8-month endovascular extension of the hybrid graft (B). FET, frozen elephant trunk.

proximal descending thoracic aorta. The distal anastomosis was performed at zone 2 of the aortic arch. An internal and external strip of Teflon felt was used to reinforce the distal anastomosis. The aortic arch reconstruction started with the reimplantation of the left subclavian artery; once completed, visceral perfusion was restored though the side branch of the prosthesis after the cross-clamping of the prosthesis in order to re-start the rewarming of the patient. The arch reconstruction was then continued by reimplanting left common carotid and innominate arteries. The procedure was finally completed with the proximal anastomosis between the supracoronary ascending aorta and the hybrid prosthesis again reinforcing the anastomosis with Teflon felt.

\section{Completion}

Subsequently, CPB was weaned off with no need for inotropic support. Operative times were as follows: $\mathrm{CPB}$ time: 209 minutes; myocardial ischemic time: 96 minutes and antegrade selective cerebral perfusion (ASCP) time: 97 minutes.

\section{Postoperative outcome}

The postoperative recovery was uneventful and the patient was discharged on postoperative day 12 . The postoperative CT scan showed a good result of the procedure.

Eight months after the procedure, the patient experienced a new episode of chest pain, radiating to the back and the CT angiogram showed the presence of an aortic dissection which started from the distal end of the endovascular portion of the Thoraflex hybrid prosthesis and extended to the external iliac arteries. The patient underwent an endovascular extension of the Thoraflex hybrid prosthesis up to the celiac trunk (Figure $2 A, B$ ).

\section{Comments}

\section{Advantages}

The use of the FET in case of arch involvement for acute type A IMH could represent a definitive treatment. The surgical technique maintains the same main steps used for type A aortic dissection. The policy in our center 
is to perform the circulatory arrest under moderate hypothermia with a continuous perfusion of both the cerebral hemispheres with a selective cannulation of the epiaortic vessels $(4,5)$. The hybrid prosthesis is selected according to the descending thoracic aortic diameter, avoiding oversizing the stented portion of the graft in cases of acute aortic syndrome and chronic dissection. Once the endograft is released we now prefer to perform the distal anastomosis in arch zone 2 to reduce the risk of spinal cord injuries and to facilitate the left subclavian artery reconstruction. Using a multibranched FET device allows for a quicker lower body reperfusion. The procedure in cases of IMH is completed in a standard fashion performing the proximal anastomosis and selective arch vessels reconstruction. We suggest reinforcing the fragile aortic wall in this type of lesions using two strips of Teflon felt inside and outside the aorta.

\section{Caveats}

Of course, close surveillance of the downstream thoracoabdominal aorta is necessary to monitor for distal progression as seen in this vignette. The stent section of the FET prosthesis is only able to cover a portion of the descending thoracic tract and therefore a further endovascular extension is often necessary during follow-up.

\section{Acknowledgments}

None.

Cite this article as: Di Marco L, Murana G, Leone A, Fiorentino M, Amodio C, Di Bartolomeo R, Pacini D. Use of the frozen elephant trunk technique for type A intramural hematoma. Ann Cardiothorac Surg 2019;8(5):574-576. doi: 10.21037/acs.2019.08.04

\section{Footnote}

Conflicts of Interest: The authors have no conflicts of interest to declare.

\section{References}

1. Czerny M, Schmidli J, Adler S, et al. Current options and recommendations for the treatment of thoracic aortic pathologies involving the aortic arch: an expert consensus document of the European Association for CardioThoracic surgery (EACTS) and the European Society for Vascular Surgery (ESVS). Eur J Cardiothorac Surg 2019;55:133-62.

2. von Kodolitsch Y, Csösz SK, Koschyk DH, et al. Intramural hematoma of the aorta: predictors of progression to dissection and rupture. Circulation 2003;107:1158-63.

3. Erbel R, Aboyans V, Boileau C, et al. 2014 ESC Guidelines on the diagnosis and treatment of aortic diseases: Document covering acute and chronic aortic diseases of the thoracic and abdominal aorta of the adult. The Task Force for the Diagnosis and Treatment of Aortic Diseases of the European Society of Cardiology (ESC). Eur Heart J 2014;35:2873-926.

4. Di Marco L, Leone A, Murana G, et al. Acute type A aortic dissection: Rationale and outcomes of extensive repair of the arch and distal aorta. Int J Cardiol 2018;267:145-9.

5. Di Marco L, Pacini D, Murana G, et al. Total aortic arch replacement with frozen elephant trunk (Thoraflex). Ann Cardiothorac Surg 2018;7:451-3. 